\title{
Intrusioni del narratore e soggettivismo nel romanzo medievale tardo: il caso del "Floriant et Florete"
}

\section{Mariateresa Prota}

\section{(2) OpenEdition Journals}

\section{Edizione digitale}

URL: http://journals.openedition.org/studifrancesi/10743

DOI: 10.4000/studifrancesi. 10743

ISSN: 2421-5856

\section{Editore}

Rosenberg \& Sellier

\section{Edizione cartacea}

Data di pubblicazione: 1 avril 2018

Paginazione: 3-13

ISSN: 0039-2944

\section{Notizia bibliografica digitale}

Mariateresa Prota, «Intrusioni del narratore e soggettivismo nel romanzo medievale tardo: il caso del "Floriant et Florete"», Studi Francesi [Online], 184 (LXII | I) | 2018, online dal 01 avril 2019, consultato il 06 janvier 2021. URL: http://journals.openedition.org/studifrancesi/10743 ; DOI: https://doi.org/ 10.4000/studifrancesi. 10743

\section{(c) (i) (9)}

Studi Francesi è distribuita con Licenza Creative Commons Attribuzione - Non commerciale - Non opere derivate 4.0 Internazionale. 


\title{
Intrusioni del narratore e soggettivismo nel romanzo medievale tardo: il caso del "Floriant et Florete"
}

\begin{abstract}
The aim of this study is to observe the narrative voice's behavior in Floriant et Florete, an arthurian romance dating back to the end of the thirteenth century, in order to see if and how it can be an index of subjectivism, have effects on the narrative structure of the work, and may provide clues as to how the work is performed. Probably, the results of the analysis may also be valid for other romances contemporary with Floriant et Florete and contribute to the framing of the evolution of the arthurian romance at the end of the thirteenth century.
\end{abstract}

Nei romanzi medievali la voce narrante extradiegetica è, come ormai assodato, al contempo eterodiegetica ${ }^{1}$, e si manifesta con maggiore intensità ed efficacia soprattutto nelle sezioni topicamente strategiche del testo, ovvero il prologo e l'epilogo - nonché, nelle opere in versi, le zone rimiche. Sono senz'altro questi, infatti, i luoghi privilegiati in cui l'istanza enunciativa s'incarna in un " $\mathrm{Io}^{2}$ e dichiara il proprio coinvolgimento nel progetto e nel compimento dell'atto compositivo, partecipando, qui più che altrove, dell'ontologia del testo letterario. Tuttavia, benché quelli appena indicati siano loci metapoetici per eccellenza e dalle dimensioni macroscopiche, non è inusuale che le intrusioni del locutore oltrepassino più volte il perimetro dei blocchi esordiali ed epilogali, sospendendo anche in zone puntiformi il normale flusso della narrazione ${ }^{3}$.

Non è un caso, dunque, che sia gli interventi iniziali e conclusivi, sia le interruzioni "in corso", benché sovente articolati secondo un repertorio piuttosto standardizzato di parole-chiave e formule, siano sottoponibili a esami puntuali e potenzialmente rivelatori dello status dell'autore e dell'identificazione del suo stile, del tipo di esecuzione del testo e delle condizioni della destinazione ${ }^{4}$ o, nei casi meno fortunati, di almeno alcuni di tali aspetti.

(1) Adotto le definizioni di G. Genette, Figures III, Paris, Seuil, 1972.

(2) Sulla presenza dell' "Io" poetico, pioneristico è lo studio di L. SPITZER, Note on the Poetic and Empirical «I» in Medieval Authors, «Traditio», IV, 1946, pp. 414-422, e illuminante quello di R. ANTONELLI, L'invenzione" dell'Io lirico, in La lirica romanza del medioevo. Storia, tradizioni, interpretazioni, Atti del VI convegno triennale della Società Italiana di Filologia Romanza (Padova-Stra, 27 settembre-1 ottobre 2006), a cura di F. BRUGNOLO e F. GAMBINO, 2009, pp. 941-953; ma di maggiore pertinenza per la ricerca che qui si propone sono le prospettive aperte da Benveniste, per il quale «je est l' individu" qui énonce la présente instance de discours contenant l'instance linguistique je»: v. E. BENVENISTE, Problèmes de linguistique générale, Paris, Gallimard, 1966, p. 251. Sempre valido, inoltre, il contributo di V. Bertolucci Pizzorusso riguardo ai risvolti letterari delle indagini sull'enunciazione nei testi medievali: v. V. Bertolucci Pizzorusso, Présence des instances du locuteur et de l'énonciateur dans les textes médiévaux, in Actes du XVIII Congrès International de Linguistique et de Philologie Romanes (Université de Trèves 1986), Tübingen, t. VI, pp. 470-479.

(3) A testimonianza del valore degli interventi della voce narrante disseminati all'interno del testo e non solo alle sue periferie, si pensi alle acquisizioni ricavate dagli studi sul narratore nel Tristan (v. E. BIK, Les interventions d'auteur dans le Tristan de Béroul, «Neophilologus» 56, I, 1972, pp. 31-42) di cui, com'è noto, il prologo e l'epilogo non ci sono pervenuti.

(4) Questo l'impegno di P. GALLAIS in Recherches sur la mentalité des romanciers français du moyen âge, «Cahiers de Civilisation Médiévale» VII, 1964, pp. 479-493 e XIII, 1970, pp. 333-347. 
Oltretutto, ampiamente superata ormai la teoria della spersonalizzazione del testo lirico medievale, le dichiarazioni della voce narrante attirano l'attenzione in quanto possibili rappresentanze di un certo coefficiente di soggettivismo insito anche in opere di carattere narrativo.

Viste la quantità e la qualità dei dati di cui si ritiene possibile il reperimento, non sarà certamente infruttuoso osservare come il narratore si manifesti nell'anonimo romanzo arturiano tardo-duecentesco di Floriant et Florete, nel quale la sua presenza è, seppure mai ingombrante, assolutamente ragguardevole. Effettivamente, degli 8.278 versi che compongono il récit, più di un terzo è occupato da monologhi e dialoghi tra i personaggi - campo d'azione, semmai, della voce narrante intradiegetica - ed è perciò altamente significativo che, dei restanti, quasi 400 siano interessati da intrusioni più o meno estese dell'istanza locutrice, le quali, vista per lo meno la loro mole complessiva, promettono di non restare silenti se sottoposte a indagine ${ }^{5}$.

Preliminarmente va osservato che l'ispezione del totale degli interventi individuati ha permesso di verificare che essi coprono l'intera gamma illustrata da Grigsby nel suo saggio a proposito della voce narrante nell'insieme dei romanzi di Chrétien ${ }^{6}$ - anticipazioni, rifiuti d'intervento, domande retoriche, opinioni, attribuzioni alla fonte, affermazioni di fedeltà al vero, asserzioni di sufficienza, proteste d'insufficienza, richiami, esortazioni/vocativi, tratti formulari e sintattici, esclamazioni ${ }^{7}$ - e la circostanza è già indicativa dell'ampiezza dello spettro di categorie funzionali messe in campo.

Le anticipazioni ${ }^{8}$, il cui costrutto tipicamente impiegato dal narratore del Floriant è «Or vous dirai de...», così come nei romanzi studiati da Grigsby fungono innanzitutto da strumento di brevità. È abitudine del nostro anonimo servirsene quasi sempre di seguito a una sospensione d'intervento, con il chiaro intento di favorire e accelerare il passaggio da una scena all'altra.

Quanto alle appena menzionate sospensioni, o rifiuti d'intervento, del tipo «Des més ne vous quier fere conte» o «Ne vous en quier fere autre fable» ${ }^{9}$, anche la loro presenza si colloca sotto il segno della concisione e della stringatezza enunciativa: evidentemente, la narrazione è sottoposta al filtraggio degli elementi considerati accessori o secondari, al fine di scongiurare il rallentamento del racconto e il ristagno

(5) I passi oggetto di studio sono stati selezionati sulla base dell'edizione più recente del romanzo (Floriant et Florete, édition bilingue établie, traduite, présentée et annotée par A. COMBES-R. TRACHSLER, Paris, Champion, 2003) e attraverso la focalizzazione delle marche formali riconosciute da Benveniste come indicative della presenza di un individuo che enuncia: pronomi e forme verbali di prima e seconda persona, deittici, verbi performativi, modo imperativo, esclamazioni, ecc. (cfr. E. BENVENISTE, Problèmes de linguistique générale II, Paris, Gallimard, 1974, pp. 79-88). Sono stati posti sotto osservazione, inoltre, i versi contenenti una terza persona, ma in cui fosse riscontrabile un cambiamento del punto di vista, riferimenti alle conoscenze generali di un ipotetico pubblico o un lessico rinviabile all'attività del raccontare.

(6) J.L. Grigsby, Narrative Voices in Chrétien de Troyes: A Prolegomenon to Dissection, «Romance Philology» XXXII, 1979, pp. 261-273; trad. it. in Il romanzo, a cura di M.L. Meneghetti, Bologna, il Mulino, 1988 , pp. $229-249$.

(7) Nelle note seguenti gli asterischi compariranno a corredo dei versi interessati da più intrusioni del narratore e perciò classificabili in diverse categorie d'intervento.

(8) Vd. i vv. 240, 576, 610, 734, 842, 946, 1193, 1254, 1629, 2016, 2612, 2744, 2776, 2887-2888, 2944, $3990,6426,6442,6878,7668,7729,7858,8083$. Un esempio a parte è costituito dal v. 56 , che sintatticamente si configura come una proposizione consecutiva, ma che dal punto di vista semantico serve ad annunciare chi sarà il protagonista del romanzo.

(9) Vd. i vv. 128, 239, 575, 733, 1253, 1628, 2015, 2089-2090, 2611, 2743, 2914, 2943, 3989, 4342, 61916192, 6255-6256, 6425, 6441, 7324-7325, 7616, 7667, 7726", 7857, 7989-7990, 8007, 8081-8082, 8120, 8123. Il v. 4342 «Du sorplus me covient taisir» è, inoltre, portatore di un plusvalore espressivo e semantico, poiché il proposito di non parlare oltre segue immediatamente la menzione delle effusioni tra gli amanti, e si configura perciò come un ammiccamento all'allocutario e un tacito invito a immaginare il non detto. 
dell'azione, peraltro rifuggiti anche attraverso altri accorgimenti espressivi, stilistici e di contenuto ${ }^{10}$.

Anche le cosiddette domande retoriche, benché rappresentate da un campione affatto folto («Que vous iroie je contant | Ne mes paroles porloignant?» vv. 2863-64; «Que diroie de leur hernois?» v. 5997; «Et por quoi lonc plait vous feroie?» v. 8174), costituiscono dei casi di variazione sul motivo della riluttanza a dilungarsi nella discussione $^{11}$, sintomo che l'aspirazione alla brevità rappresenta per il narratore un vero e proprio programma di lavoro.

Le opinioni e i pareri, del tipo «ce m'est avis» o «com il me samble» ${ }^{12}$, nella maggioranza dei casi funzionano come riempitivi di interi emistichi - solitamente i secondi - e la loro natura di zeppe, oltre che dall'aspetto formulare, è comprovata dalla posizione ravvicinata, e quindi pleonastica, di alcune di esse, nonché, talvolta, dalla poca congruenza rispetto al contesto. C'è da credere, forse, che l'autore non percepisca tanto il valore locutivo di tali inserti, quanto la loro appartenenza a un rimario facilmente accessibile e a un repertorio di forme spendibili come sostegni architettonici nella costruzione dell'edificio narrativo. Un impiego del genere, comunque, non determina un insuccesso estetico: se, infatti, le zone rimiche possiedono un'importanza fonetica e semantica, nei casi che ci occupano il valore poetico di una sonorità che si ripete e si riverbera, quasi si trattasse di un refrain, non può essere taciuto. L'autore doveva certamente compiacersene, visto il ricorrere frequente nel dettato sia di espedienti sonori di tal fatta sia di figure etimologiche e dei più comuni procedimenti di allitterazione ${ }^{13}$.

Le attribuzioni a una fonte ${ }^{14}$ - per esempio «Si com l'estoire nous devinne» - non sono numerosissime e compaiono tutte nell'ultimo terzo del testo. La loro concentrazione nella parte terminale dell'opera, senza alcuna allusione a un'autorità in quella esordiale, solleva qualche sospetto sull'effettiva esistenza di un precedente racconto; per giunta le caratteristiche di assoluta genericità e, talvolta, l'identità di versi che contraddistinguono tali attribuzioni, sembrano qualificarle come semplici espedienti per la legittimazione di un racconto non solo già avviato, ma prossimo alla conclusione.

Le affermazioni di aderenza al vero nel Floriant risultano spesso sovrapponibili alle dichiarazioni di sufficienza, e sono perciò a queste assimilate nella nostra rassegna. Le espressioni tipiche di questa doppia categoria sono «Si com je vous cont»o «Mentir ne vous en quier». Esse, peraltro, sono senza dubbio degne di nota per la frequenza con cui ricorrono ${ }^{15}$ e per la loro distribuzione piuttosto omogenea lungo tutto il testo, sebbene, d'altra parte, l'aspetto formulare di tali passaggi sia innegabile.

(10) Si condivide pienamente la definizione di fiction pressée attribuita al Floriant dai suoi ultimi editori, secondo i quali «ce roman dévore véritablement le temps». Serve a questo obiettivo, per esempio, l'uso frequente del passato prossimo e degli avverbi di tempo indicanti la repentinità degli avvenimenti. Si veda A. COMBEs-R. TRACHSLER (éds), op. cit., pp. LII-LVI.

(11) Dei tre esempi individuati, il primo e l'ultimo rientrano esplicitamente nelle considerazioni fatte, il secondo può comunque essere interpretato come una variante del tipo osservato, visto il carattere sintetico della risposta retorica che introduce.

(12) Vv. 18, 46, 304, 472, 516, 663, 766, 842, 910, 958, 961, 1140, 1192, 1217, 1263, 1359, 1361, 1454, 1532, 1568, 1934, 2124, 2195, 2238, 2280, 2321, 2428, 2456, 2496, 2667, 2746, 2867, 2958, 3406, 3758, $3761^{*}, 3764,3777,3868,4358,4364^{\prime \prime}, 4686,4757,5048^{*}, 5081,5133,5250,5274,5384,5446,5782,5971$, 5987, 5992, 6110, 6135, 6148, 6155, 6333, 6416, 6578, 6736, 6738, 7676, 7726", 7834, 7861, 7882, 8006, 8193.

(13) Lo si può appurare agevolmente attraverso una lettura attenta dell'intero romanzo.

(14) V. i vv. 6082, 6452, 6586, 7728, 7992, 8008.

(15) V. i vv. 138, 292, 928, 1554-1555, 1632, 2125, 2132, 2134, 2597, 2639, 2672, 2906, 3242, 3680, $5048^{\prime \prime}, 5138,5240,5928,6803-6804,6014,6092,6099,6107,6126,6194,6280,6310,6418,6763,7021$, 
Quanto alle poche proteste d'insufficienza ${ }^{16}$, come «Nel vous puis pas toutes nomer | Ne je ne savroie nombrer», non sembrano attenere tanto alla topica della modestia - cui ricorrono di prassi anche gli autori più illustri e affermati - quanto all'intenzione di esaltare la straordinarietà di un oggetto ineffabile o la cui descrizione richiederebbe troppo tempo per poter essere intrapresa e portata a compimento.

Sulla scia di tali interventi sono collocabili anche le costruzioni iperboliche del tipo «onques plus riche ne vit home», «onc mius ovree ne fu», ecc. ${ }^{17}$, le quali, oltre a indicare l'eccezionalità di qualcuno o di qualcosa, rimandano all'orizzonte di conoscenze degli allocutari, parametro per l'effettiva valutazione del grado di unicità presunta. Esse, pertanto, sembrano configurarsi parimenti come espedienti per l'interlocuzione con i destinatari dell'opera, tanto più che, nel nostro romanzo, l'espressione iperbolica nei casi più numerosi ricorre nelle vicinanze di un'esplicita affermazione del narratore con marche formali di prima persona singolare.

I richiami, come quello al v. 965 «Touz ciaus que je vous ai nomez» ${ }^{18}$, sono registrati in numero relativamente cospicuo. Il narratore di Chrétien, al contrario, è piuttosto restio a questo tipo d'intervento, probabilmente perché il raffinato romanziere di Troyes avvertiva l'origine epica di una tale tecnica, che difatti, nei suoi sporadici impieghi, relega in associazione agli elenchi di nomi ${ }^{19}$. Nel nostro romanzo un uso del genere compare soltanto due volte su otto, mentre nei casi restanti esso è utile a riportare alla memoria del pubblico un personaggio nominato in precedenza o, ancor di più, a focalizzare ulteriormente l'attenzione su ciò di cui si sta parlando al momento: una sorta di deriva della tecnica del richiamo verso la richiesta d'ascolto.

$\grave{E}$ da segnalare, poi, la presenza notevole di esortazioni e vocativi ${ }^{20}$, in buona parte inerenti la sfera semantica del sentire/ascoltare, sebbene talvolta in forma attenuata: «Se vous entendre me voulez».

Alla categoria delle espressioni formulari e sintattiche ${ }^{21}$ appartengono i tipi «es vous», «vessiez», «oïssiez», in cui il pronome e la forma verbale alla seconda persona plurale rinviano a un destinatario altrettanto plurale ${ }^{22}$, mentre l'avverbio "es", dalla forte connotazione attualizzante, è impiegato per introdurre l'arrivo sulla "scena" di un nuovo personaggio.

Le esclamazioni ${ }^{23}$ - siano esse di amarezza, di sorpresa o sentenziose - sono disseminate lungo tutto il testo e, salvo pochi $\operatorname{casi}^{24}$, non sembrano riducibili a meri

7180, 7727, 7754*, 7832, 7860, 7984, 8124. Può essere classificata in questo gruppo anche la locuzione «mien esciant», «par le mien esciant» (cfr. i vv. 117, 845, 5963, 6098, 6127, 7848) traducibile con "sulla mia fede", ma anche con "a mio avviso" o "per quel che ne so". Ë, pertanto, un' espressione di fedeltà che presenta, però, notevoli connivenze con l'idea del giudizio personale e della propria conoscenza dei fatti; in quest'ultimo caso, comunque, sembra azzardato che si tratti di un esplicito rinvio a una fonte, vista proprio la versatilità della locuzione.

(16) Vv. 2669-2670, 3082, 5276, 6798-6802, 7020.

(17) Cfr. i vv. 108, 110-113, 126-127, 844-845, 903, 935, 1214, 1262-1263, 2498, 2673-2674, 2865-2868, 2885-2886, 2908, 3992, 4559-4560, 5241, 5998-5999, 6016-6017, 6797, 6804, 6949, 7985-7988, 8124-8125.

(18) Ma v. anche i vv. 735, 909, 1453, 2921, 4663, 6408, 6771.

(19) Si veda a tal riguardo il saggio già citato di J.L. GRIGSBY, Narrative Voices in Chrétien de Troyes.

(20) Vv. 231-232, 450, 970, 1194, 1490-1491, 1537, 1685*, 2212, 2522, 2800, 3768, 4309, 4364", 5040, $5563,5839,6230,6998-6999,7646,8276$.

(21) Vv. 1836, 2408, 2458, 2631, 2702, 3339, 3541, 3627, 3761", 3850, 3852, 4677, 4746, 5037, 5354, 5483, 5494, 6116, 6117, 6125, 6218, 6332, 6465, 6546, 7140, 7485, 7486, 7492, 7502, 7754", 7963, 7981, $8075,8272$.

(22) Pari valore presentano anche le forme alla terza persona singolare del tipo «qui veïst» (cfr. i vv. 302 , $1555-1556,2301,2415,3870,3872$ ) in cui il pronome indefinito rimanda all'esperienza che l'allocutario avrebbe potuto vivere se si fosse trovato nella situazione descritta.

(23) Vv. 20-21", 221-222, 294-295, 338-339, 350, 363, 1290-1291, 1307-1308, 1685", 2138, 2141, 2458, 2492, 3732, 3765, 3768, 3939-3940, 4359-4364", 4365-4368, 4371, 4438, 5810-5811, 6102, 7144.

(24) Il tipo «se Diex me gart», presente ai vv. 2138, 2458, 2492, 3765, 4438, tra quelli di nostro inte- 
clichés. Si tratta, in effetti, di interventi perfettamente integrati nel contesto narrativo e non caratterizzati da ripetitività di contenuto e di espressione, circostanza peraltro in contrasto significativo con la formularità generalmente riconosciuta come cifra stilistica del nostro romanzo ${ }^{25}$.

Un'espressione esclamativa, del resto, si registra anche nel prologo ${ }^{26}-\operatorname{luogo} \mathrm{di}$ cui si è già menzionata la crucialità - e se la sua presenza qui non è eccezionale, forse è meno comune il suo tono appassionato:

Molt voi de gent qui rimer voelent [...]

Mes molt se doit bien porpenser

Qui s'entente a rimer velt metre

Qu'il s'en sache bien entremetre

Qu'il puist rimer en tel manier

Que par devant ne par derriere

Ne soit gabez ne escharnis.

Quar Mesdires, ce m'est avis,

A molt du siècle en sa baillie.

Mesdisant, Jhesu vous maudie

Et vous doint la male aventure!

Quar je n'ai de voz mesdis cure

$\mathrm{Ne}$ ja por vous ne quier laisser

Ma matere a encommencier.

(vv. 9, 12-24)

Il contenuto del passaggio in esame è quanto mai topico: i maldicenti sono consueti destinatari degli improperi dei poeti cortesi e l'anatema è introdotto da una tradizionale riflessione sull'abilità nel comporre versi. Tuttavia l'invettiva, malgrado il suo carattere non inedito, risulta tanto violenta quanto inaspettata, visti la pacatezza e l'andamento "ragionato" del discorso precedente, e pare qualificarsi come apostrofe enfatica realmente funzionale soltanto se impiegata in un contesto orale e drammatizzante, in cui la dichiarazione di ostilità nei confronti di una precisa categoria - che probabilmente l'autore immagina come un gruppo astratto di potenziali detrattori - accresce per converso il valore del "nous" iniziale ( «Salemons nous dist que tant est | Li fols sages com il se test», vv. 1-2) con cui si sottolinea l'appartenenza del locutore allo stesso orizzonte culturale degli allocutari.

Certamente, nulla consente di escludere che tali strutture enfatiche costituiscano degli artifici retorici predefiniti, ma pur tenendo in considerazione il loro probabile carattere non estemporaneo, è possibile che essi siano congegnati allo scopo preciso di favorire l'interazione dinamica con il pubblico: espedienti, quindi, per vivacizzare la lettura e conferirle un tono spettacolare.

A tal proposito, più volte è stato riconosciuto e ribadito dagli studi il dominio dell'oralità ${ }^{27}$ nella cultura medievale in generale e nella sua produzione letteraria in particolare, benché tale aspetto inizi lentamente ad attenuarsi a partire dal XIII secolo;

resse, sembra essere usato come riempitivo dell'emistichio sia dal narratore extradiegetico che da quello intradiegetico.

(25) Si veda almeno A. Combes-R. TRAChsler (éds), op. cit., pp. LXVI-LXVII.

(26) Del prologo del Floriant come esempio di tipo "lirico" si discute in S. MENEGALDO, Quand le narrateur est amoureux: prologues et épilogues liriques dans le roman de chevalerie en vers au XII et XIII siècles, «Bien Dire et Bien Aprandre» 19, 2001, pp. 149-165.

(27) Tra i numerosi studi sull'argomento, si veda almeno l'imprescindibile saggio di P. ZumTHOR, La lettre et la voix. De la «littérature» médiévale, Paris, Seuil, 1987; trad. it. La lettera e la voce. Sulla «letteratura» medievale, a cura di M. Liborio, Bologna, il Mulino, 1990. 
comunque, è assodato che ancora nei testi databili entro la metà del Duecento, gli interventi della voce narrante abbiano prioritariamente una funzione situazionale, di ancoraggio dell'opera all'bic et nunc, dato il perdurare dell'esecuzione orale a quel tempo ${ }^{28}$.

Il nostro romanzo, invece, è stato ascritto agli ultimi decenni del XIII secolo ${ }^{29}$, ma la presenza abbondante di allocuzioni, esortazioni ed espressioni rinvianti alla sfera sensoriale dell'udito dimostra la sua appartenenza a un ambiente culturale in cui la lettura ad alta voce - se non addirittura la recitazione - del testo letterario per una cerchia di ascoltatori rappresenta una pratica ancora affatto dismessa, o per lo meno minimamente inficiata dall'allora nascente lettura silenziosa, attività progressivamente affermatasi nel secolo successivo ${ }^{30}$ e con la quale la performance orale dovette certamente convivere a lungo. Peraltro, proprio l'impiego attualizzante, osservato in precedenza, dei tratti formulari e sintattici e dei richiami suffraga l'ipotesi di un'esecuzione in pubblico e denota, quindi, il persistere del carattere teatralizzante della narrazione.

È possibile, ovviamente, che a quest'altezza le locuzioni appena citate rappresentino soltanto dei retaggi, ormai cristallizzati, di sintagmi in auge nel passato, espressioni passepartout facili da reperire e adattare, per di più legate da un fil rouge alla tradizione. Tuttavia, quanto riferito nel seguente passaggio di metapoetica:

D'autre part sont cil conteour,

La est des chevaliers la flour

Quar volentiers les escoutoient

Que les anciens faitz contoient

Des preudomes qui jadis furent

Qui se maintinrent si com durent,

Des grant batailles que il firent

Et comment lor terre conquirent.

Tout ce li conteeur contoient

Et il volentiers les ooient

Et se miroient es biaus dis,

S'en devenoient mieux apris.

Quar qui romanz velt escouter

Et es biaus dis se velt mirer

Merveil est s'il ne s'en amende

S'il est ensi qu'il i entende: [...]

(vv. 6231-6246)

conferma inequivocabilmente l'idea - almeno nell'immaginario dell'autore - di una fruizione auricolare e collettiva del testo letterario.

Peraltro, e quasi paradossalmente, che l'utilizzo fatto dal narratore delle allocuzioni e degli incitamenti all'ascolto non sia meramente, o esclusivamente, il frutto del

(28) P. Gallais, Recherches cit., 1964, pp. 483-484; P. Zumthor, Essais de poètique médiévale, Paris, Seuil, 1972, pp. 37-38.

(29) Per una bibliografia v. A. Combes-R. TRAchsler (éds.), op. cit.; mi permetto di rinviare, inoltre, al mio contributo Il valore dell' intertestualità nella datazione del Floriant et Florete, in «Medioevo Romanzo», 40, 2, 2016, pp. 427-441.

(30) Seppur rivolta specialmente alla poesia lirico-narrativa, è di particolare interesse per le acquisizioni estensibili anche ad altri generi letterari l'indagine condotta da Sylvia Huot a proposito del passaggio, a suo avviso avvertibile proprio tra la fine del XIII secolo e l'inizio del XIV, dalla declamazione alla lettura e dal concetto di libro come registrazione di una performance orale a prodotto di scrittura. Si veda S. HUOT, From Song to Book: The Poetics of Writing in Old French Lyric and Lyrical Narrative Poetry, Ithaca-London, Cornell University Press, 1987. 
reimpiego di frasi fatte, ma risponda alla reale esigenza di mantenere alto l'appeal del racconto, è testimoniato dalla loro assoluta mancanza nel prologo ${ }^{31}$. È stato, infatti, rilevato come il raffinato pubblico di corte, educato all'ascolto dei romanzi, non necessitasse di particolari sollecitazioni a prestare attenzion $\mathrm{e}^{32}$, soprattutto all'inizio di una lettura certamente caldeggiata; nel prosieguo, invece, il fisiologico calo di concentrazione poteva essere abilmente evitato attraverso invocazioni cadenzate all'uditorio.

Proprio a proposito del prologo, lascia certamente delusi l'assenza di tutti quegli elementi che spesso trovano spazio nei versi incipitari e che, quando l'anonimato di alcuni testi medievali si lascia minare, sono in grado di fare luce sull'identità dell'autore: non è concesso di rinvenire, infatti, alcun riferimento alla passata produzione di quest'ultimo, non un'allusione a un mecenate, non un toponimo che funga da indicazione d'origine, nessuna attribuzione a una fonte e nessun tentativo di accreditamento $^{33}$. Lo stesso vale per l'epilogo, addirittura sprovvisto di una reale corposità, essendo ridotto a soli tre versi in cui il narratore assicura che nulla si seppe mai più dei due protagonisti e afferma di voler terminare in quel modo il proprio racconto $(«[\mathrm{~S}]$ achiés des ici en [avant] | [N]'oÿ nus hon d'iaus.IJ. p[arler] | Si voldrai mon conte fi[ner]», vv. 8276-8278).

A ogni modo, malgrado l'assenza d'informazioni certe, dall'esame complessivo degli interventi del narratore è possibile per lo meno inferire indizi a proposito dell'esperienza del nostro autore nel campo delle lettere e della sua probabile condizione di esordiente.

Innanzitutto, che il nostro anonimo sia forse un romanziere novizio è in linea con la sua loquacità, che senz'altro agevola nell'organizzazione del materiale narrativo, ed è testimoniato dall'impiego proprio in tal senso di specifiche intrusioni dell'istanza locutrice. Tra queste rientrano in primo luogo le anticipazioni, il cui utilizzo a seguito di un rifiuto d'intervento ne fa dei veri e propri giunti di transizione, evidentemente rudimentali, e infatti progressivamente abbandonati lungo la narrazione: nella prima metà del dettato si contano il doppio delle anticipazioni rispetto alla seconda, come se la penna dell'anonimo si andasse lentamente affinando, prediligendo trapassi più ricercati.

Indubbiamente, è indicativo di una coerenza di comportamento e di una pianificazione d'intenti il fatto che anche tutti gli altri procedimenti che sono stati visti essere rivolti a favorire la brevità narrativa - i rifiuti d'intervento, le domande retoriche, le proteste d'insufficienza - possano essere posti al servizio delle esigenze strutturali, in virtù della loro spendibilità quali elementi di snodo nel montaggio di scene e episodi.

È da intendersi allo stesso modo l'uso pleonastico delle opinioni, come già detto valutabili nella doppia funzione di zeppe e di rimanti immediati, cui poteva ricorrere una penna dilettantesca in cerca di facili congiunture. A latere c'è da aggiungere, comunque, che un impiego del genere certamente non collide con il probabile carattere orale dell'esecuzione, visto che proprio formule e ripetizioni costituiscono le cifre stilistiche per eccellenza di componimenti - le canzoni di gesta, per esempio - pensati non per la semplice lettura ad alta voce, ma addirittura per la recitazione.

Spie di un autore esordiente sono ricavabili anche dalle affermazioni di aderenza al vero e dalle dichiarazioni di sufficienza, non tanto per il loro apporto contenuti-

(31) Dall'analisi statistica condotta da Gallais (cfr. Recherches cit., 1964, p. 489) è emerso che all'interno dei prologhi le allocuzioni al pubblico nella forma dei verbi oyez, escoutez, entendez, sono presenti nel $50 \%$ delle canzoni di gesta, nel $40 \%$ delle vite dei santi e nel $35 \%$ dei romanzi.

(32) Ibidem.

(33) Come visto sopra, comunque, le ultime due categorie d'intervento menzionate non sono prive di rappresentanza nel resto del romanzo. 
stico, quanto perché sembrano evidenziare le ansie di un locutore poco navigato, realmente bisognoso di sentirsi rassicurato nei propri mezzi o di mascherare qualche incertezza ribadendo al pubblico costante onestà narrativa.

Oltre alle categorie d'intervento finora illustrate, e come già detto rappresentate anche nei testi cristaniani, merita di essere messa in luce come circostanza non molto comune nel genere romanzesco, l'inclinazione del Floriant a fare spazio a brani di puro soggettivismo. A tal proposito, passo certamente emblematico - e, peraltro, utile anche a misurare la reale portata delle già menzionate esclamazioni - è il vistoso intervento "al mezzo" nel quale il locutore dichiara la propria condizione d'innamorato, prima ricambiato e poi respinto:

Je ne pens pas qu'il lor anuit

Quar se lez m'amië estoie

Et une nuit i demouroie

Et la nuit .IIIJ. jors durast

$\mathrm{Ne}$ devant donques n'ajornast

Que le sinquieme entrez seroit,

Sachiés que poi me sambleroit!

Dex! porroit il mais avenir

Que je la poïsse tenir

Trestoute nue entre mes braz?

Nenil, voir, e mi! chaitis, laz!

J'ai veü que j'estoie amez,

Mes or sui arrieres boutez,

Ce fet Fortune desloiaux! [...]

(vv. 4358-4371)

In questo punto, di fatto centrale, l'interruzione della narrazione rievoca - come già segnalato dagli ultimi editori del Floriant ${ }^{34}$ - le più note intrusioni dell'istanza locutrice nei romanzi di Partonopeu de Blois, Le Bel Inconnu e Joufroi de Poitiers, osservate attraverso la lente strutturalista da Grigsby ${ }^{35}$ sul finire degli anni Sessanta.

Per i narratori delle tre opere appena citate, l'ispirazione sostanziale, e apertamente dichiarata nel prologo, risiede nel soddisfacimento dei desideri della propria amata, tant'è vero che talvolta il racconto rischia addirittura di arrestarsi a causa dello scontento della dama ${ }^{36}$. Visto, quindi, il ruolo di principio ispiratore assunto dagli amori dei narratori, le varie affermazioni di questi ultimi a proposito della loro situazione sentimentale risuonano come echi autorizzati da un disegno narrativo circola$\mathrm{re}^{37}$. Nel nostro romanzo, invece, veniamo a malapena informati dell'esistenza di una dama amata dal locutore grazie a un'invocazione esordiale ( $\ll \mathrm{Et}$ ausi la debonnere | Qui a mon cuer en sa baillie | Dont ja n'en partira ma vie», vv. 28-30) - topica e telegrafica tanto quanto quella canonica ad Amore («Voeil proier Amors que s'aïe I M'ostroit a ceste chose fere», vv. 26-27), al quale si chiede esplicitamente aiuto nel comporre - ma la donna è dimenticata nell'epilogo, e la sua approvazione o il suo rifiuto non sembrano avere incidenze significative sul buon esito della narrazione.

(34) Si veda A. Combes-R. Trachsler (éds), op. cit., p. 263, nota 1.

(35) J.L. Grigsby, The Narrator in "Partonopeu de Blois", "Le Bel Inconnu" and "Joufroi de Poitiers", «Romance Philology» XXI, 1968, pp. 536-543.

(36) Ibidem, p. 538.

(37) In particolare a proposito del Partonopeu, Lori Walters ha parlato delle esternazioni di tono lirico del narratore quali strumenti per lo sviluppo narrativo: L. WALTERS, The poet-narrator's address to bis lady as structural device in "Partonopeu de Blois", «Medium Ævum» 51, 1992, pp. 229-241. 
Non è senza significato, in proposito, che, a metà del testo, il nuovo riferimento all'amata non abbia alcuna interferenza rispetto al prosieguo dell'opera e all'attività del poetare.

La confessione del narratore, insomma, indotta per contrasto dalla vicenda dei protagonisti e peraltro traboccante di sentimento e passione, è disgiunta dalle precedenti dichiarazioni e ha il valore della parentesi incidentale: il suo significato si esaurisce nel blocco versale occupato, senza riverberarsi nel resto del dettato. Al contempo, però, essa ha il potere abbagliante del flash che non può che catturare l'attenzione degli ascoltatori e mantenere saldo il canale di comunicazione con loro instaurato.

La circostanza appena menzionata non è isolata: si contano, infatti, altri due casi in cui la voce narrante si dilunga in interventi accorati, in cui le vicende dei personaggi inducono a riferimenti a conoscenze proprie o esperienze personali. Accade ai vv. 221-238, poco prima dell'uccisione del re di Sicilia Elyadus da parte del suo siniscalco Maragot:

He las! Com c'est grant mesprison

Et com vilainne traïson!

Mes ja sovent oï retraire

C'onques hons sers ne pot bien faire.

Li rois Daires en fu murtris

Et Julius Cesar occis

Et Alixandre empoisonnez

Et li rois Pepins enherbez

Et Charles ses filz dechaciez

Et Elyadus detrenchiez,

Se vous entendre me voulez,

Si com par tanz oïr pourrez.

Bien sai qu'il lor vient de nature

Qu'il sont traïtor par droiture:

Quaïns fu leur anciens peres,

Cil qui ocit .J. de ses freres,

Encor dure leur felonnie,

Leur fausseté, leur tricherie

e ai vv. 2587-2610, in cui il motivo topico del declino dei tempi è trattato con estrema partecipazione:

Dont ert li siecles de bon point

Mes orendroit n'i a il point

De raison ne de loiauté:

Tot voi le bien a mal torné,

Loiautez a noient s'atorne,

Desloiautez le mont ajorne.

Nus n'aime mais la loiauté,

Tuit servent la desloiauté,

Et avarice et mauvestié

Est bien creüe la moitié.

Tant vous puis pour verité dire

Que cist siecles tozjor empire.

Empire, oil! de toutes pars,

Li bien est perdus et espars

Et li maux est monteplié.

A mal se sunt tuit ravoiez:

Li baron de plusors contrees

Tuit ont mes lor mesons hordees 
De Fauseté, de Tricherie,

De Traïson, de Roberie,

De Destrece, de Couvoitise

Qui molt de maux bersse et atisse.

Ensi li siecles mesdevient,

Par force avenir le covient!

Sicuramente è indicativo di un modus operandi che il narratore si compiaccia nel riservarsi degli spazi di autonomia per estrinsecarsi con particolare pathos - peraltro rasentando anche qui la teatralità perseguita altrove attraverso altre categorie d'intervento - ma è bene segnalare che tale aspetto fa sì che il Floriant rientri tra le opere protagoniste del movimento di avvicinamento della letteratura duecentesca al discorso sul soggetto; un processo che si compie generalmente nella lirica attraverso l'invasione di riferimenti biografici e narrativi responsabili del passaggio dalla canzone al dit, nel romanzo grazie a spaccati di effusione lirica e a un tipo di narrazione progressivamente pensata per essere letta individualmente piuttosto che declamata ${ }^{38}$.

È ormai assodato, infatti, che, nel campo di cui ci occupiamo, ovvero la narrazione, il lirismo e le dichiarazioni d'amore del narratore alla propria dama rappresentino nel XIII secolo una consuetudine sempre più frequente ${ }^{39}$ e dagli inevitabili risvolti sia sul piano strutturale che su quello contenutistico: si pensi al precoce, e in tal senso avanguardistico, Partonopeu de Blois ${ }^{40}$; al Roman de la rose, atto di nascita del romanzo allegorico; o al caso particolarissimo del Roman du Chastelain de Coucy et de la dame de Fayel, congegnato da Jakemes con l'intento di fornire una cornice biografica alle liriche del troviero ${ }^{41}$.

Il nostro récit, invece, si distingue per il fatto che, se negli altri rappresentanti romanzeschi del cambiamento l'irruzione della soggettività è tale da consentire di tracciare una linea biografico-sentimentale dell'autore/narratore quasi parallela a quella narrativa oppure di sfruttare i passaggi lirici come dispositivi strutturali, nel Floriant et Florete i luoghi in cui la soggettività si affaccia sono slegati tra di loro, solo in un caso di argomento amoroso, sciolti da vincoli strutturali e, come già detto, non anticipati o seguiti da alcuna informazione di carattere biografico nel prologo e nell'epilogo.

Tale circostanza non è secondaria, al contrario, proprio per la sua eccezionalità, merita adeguato rilievo e, perlomeno, un tentativo d'interpretazione. Difatti, la schiettezza e gratuità degli slanci patetici potrebbero pure costituire un'ulteriore prova dell'inesperienza del nostro narratore, incapace di eclissarsi totalmente dietro la vicenda dei propri protagonisti e incline a "conversare" per far fronte a difficoltà o aggirare ostacoli sul piano dell'articolazione dell'opera; tuttavia, l'utilizzo di un procedimento, ormai divenuto di moda tra i romanzieri, proprio in luoghi del testo in cui la sua presenza non pare indispensabile ai fini strutturali, dimostra che nel Floriant et Florete il soggettivismo non è avvertito come un aspetto da giustificare nell'architettura dell'edificio narrativo, ma come un elemento naturalmente appartenente alla narrazione stessa. In sostanza, proprio nel nostro romanzo, d'impianto tradizionale,

(38) Si veda in proposito il saggio illuminante di MichEL ZINK: La subjectivité littéraire. Autour du siècle de saint Louis, Paris, Presses Universitaires de France, 1985. La questione è stata ripresa più tardi da Charmaine LeE nel suo La soggettività nel Medioevo, Roma, Vecchiarelli Editore, 1996.

(39) D. QuÉRUEL, Roman d'amour et déclaration d'amour: une nouvelle mode romanesque à l'aube du XIII siècle, «Travaux de littérature» 10, 1997, pp. 33-48.

(40) Si veda L. WALTERS, op. cit.

(41) Per gli ultimi due esempi menzionati si vedano le interessanti osservazioni di S. HuOt, op. cit., pp. 83-105 e 117-134. 
ancorato all'idea di esecuzione pubblica dell'opera letteraria e rudimentale nell'utilizzo di talune tecniche di costruzione testuale, il discorso sul soggetto o del soggetto sembra essere condotto al più alto grado di maturità.

A conclusione della disamina esperita a proposito della voce narrante nel Floriant sono emerse tre circostanze interessanti e dai risvolti potenzialmente estensibili ad altre opere coeve alla nostra: in primo luogo, il perdurare, nell'ultimo scorcio del XIII secolo, della pratica della lettura ad alta voce dei romanzi cortesi, che il pubblico ancora doveva ascoltare come si trattasse di una performance da cui trarre diletto collettivamente; in secondo luogo, il valore strutturale e tecnico di alcune intrusioni del locutore, sfruttate quali puntelli immediati ma d'effetto per facilitare l'organizzazione del materiale narrativo, e al contempo in linea con l'impostazione teatralizzante dell'opera; infine la presenza di una buona quota di soggettivismo, aspetto in linea con i nuovi esperimenti della letteratura duecentesca, ma trattato nel Floriant in maniera autonoma e innovativa. 